New Theory

ISSN: 2149-1402
37 (2021) 86-98

Journal of New Theory

https://dergipark.org.tr/en/pub/jnt

Open Access

\title{
On the Existence of Symmetric Positive Solutions for SSBVPs on Time Scales
}

\author{
Cansel Kuyumcu $^{1}{ }^{\mathbb{D}}$, Erbil Çetin ${ }^{2}$
}

\author{
Article History \\ Received: 13 Dec 2021 \\ Accepted: 29 Dec 2021 \\ Published: 31 Dec 2021 \\ 10.53570/jnt.1036239 \\ Research Article
}

\begin{abstract}
This paper considers symmetric positive solutions for the system of second-order boundary value problems (SSBVPs) on time scales using the Krasnosel'skii fixed point theorem. We then demonstrate two examples. Finally, we discuss the need for further results.
\end{abstract}

Keywords - Time scale, symmetric positive solution, boundary value problem, Krasnosel'skii fixed point theorem

Mathematics Subject Classification (2020) - 34K42, 34A05

\section{Introduction}

Hilger [1] first proposed the concept of time scales. The time-scale approach, used to model phenomena that manifest partly in discrete-time and continuous-time, unifies difference and differential equations. [2-5] provide heat transfer, stock market, economic, epidemic models and biological by utilising this concept. There has been an appreciable interest and research about boundary value problems on time scales in the past two decades. Moreover, there have been many results recently about the existence and multiplicity of symmetric positive solutions (SPSs) for nonlinear second-order and higher-order differential and dynamics equations with boundary conditions [6-14].

In [6] Qu investigated existence of positive solutions for following second order differential equations

$$
\begin{aligned}
& -u^{\prime \prime}(t)=f(t, v), \\
& -v^{\prime \prime}(t)=g(t, u), \quad t \in[0,1]
\end{aligned}
$$

with following boundary conditions

$$
\begin{aligned}
& u(t)=u(1-t), \\
& u^{\prime}(0)-u^{\prime}(1)=u\left(\xi_{1}\right)+u\left(\xi_{2}\right) \\
& v(t)=v(1-t) \text {, } \\
& v^{\prime}(0)-v^{\prime}(1)=v\left(\xi_{1}\right)+v\left(\xi_{2}\right)
\end{aligned}
$$

where $0<\xi_{1}<\xi_{2}<1$.

Inspired by the studies mentioned above, we will study the existence of SPSs for the following system of second-order boundary value problems (SSBVPs).

\footnotetext{
${ }^{1}$ canselkuyumcuuu@gmail.com; ${ }^{2}$ erbil.cetin@ege.edu.tr (Corresponding Author)

${ }^{1,2}$ Department of Mathematics, Faculty of Sciences, Ege University, İzmir, Turkey
} 


$$
\begin{cases}-\vartheta^{\Delta \nabla}(t)=f(t, \varphi), & t \in \mathbb{T}_{\kappa}^{\kappa} \\ -\varphi^{\Delta \nabla}(t)=g(t, \vartheta), & t \in \mathbb{T}_{\kappa}^{\kappa}\end{cases}
$$

subject to the boundary conditions

$$
\left\{\begin{array}{rlrl}
\vartheta(t) & =\vartheta(b-t+a), & & \alpha\left[\vartheta^{\Delta}(a)-\vartheta^{\Delta}(\rho(b))\right]=\vartheta\left(\tau_{1}\right)+\vartheta\left(\tau_{2}\right) \\
\varphi(t)=\omega(b-t+a), & & \alpha\left[\varphi^{\Delta}(a)-\varphi^{\Delta}(\rho(b))\right]=\varphi\left(\tau_{1}\right)+\varphi\left(\tau_{2}\right)
\end{array}\right.
$$

where $\mathbb{T}$ is a bounded symmetric time-scale (with $a=\min \mathbb{T}$ and $b=\max \mathbb{T}$ ), $\tau_{1}, \tau_{2} \in \mathbb{T}$ such that $\tau_{1}=b-$ $\tau_{2}+a, \alpha \geq b-a$ and $f, g: \mathbb{T} \times \mathbb{R}^{+} \rightarrow \mathbb{R}^{+}$are $l d$-continuous functions, both $f(., s)$ and $g(., s)$ are symmetric on $\mathbb{T}$ time-scale such that $f(t, 0) \equiv g(t, 0) \equiv 0$.

The second section of the present paper provides some of the basic definitions and lemmas needed for the next sections. Section 3 proves SPSs for the system (1)-(2) using the Krasnosel'skii fixed point theorem and presents two examples to illustrate the main results herein. Finally, we discuss the results and future studies. This study is a part of the first author's master's thesis.

\section{Preliminaries}

This section presents some basic definitions and results concerning about time scale theory which can be in [15-17]. Let $\mathbb{T}$, a nonempty closed subset of $\mathbb{R}$, be a time scale such that $a=\min \mathbb{T}$ and $b=\max \mathbb{T}$. The jump operators $\sigma, \rho: \mathbb{T} \rightarrow \mathbb{T}$ are defined by $\sigma(t):=\inf \{s \in \mathbb{T}: s>t\}, \rho(t):=\sup \{s \in \mathbb{T}: s<t\}$ where $\inf \varnothing=a$ and $\sup \emptyset=b$ so that $\rho(a)=a$ and $\sigma(b)=b$. A point $t \in \mathbb{T}$ is called right scattered, right dense, left scattered, and left dense if $\sigma(t)>t, \sigma(t)=t, \rho(t)<t$ and $\rho(t)=t$ respectively.

If a function $x$ from $\mathbb{T}$ to $\mathbb{R}$ is continuous at all left dense points and has finite right-sided limits at all right dense points of $\mathbb{T}$, then it is said to be $l d$-continuous on $\mathbb{T}$. Moreover, $\mathcal{C}_{l d}(\mathbb{T})$ denotes the set of $l d$-continuous functions $x: \mathbb{T} \rightarrow \mathbb{R}$. Here, $\mathcal{C}_{l d}(\mathbb{T})$ is a Banach space with below norm $\|x\|:=\max _{t \in \mathbb{T}}|\mathrm{x}(\mathrm{t})|, x \in \mathcal{C}_{l d}(\mathbb{T})$.

Throughout this paper, let define the delta and nabla differentiability sets $\mathbb{T}^{\kappa}:=\mathbb{T}-(\rho(b), b], \mathbb{T}_{\kappa}:=$ $\mathbb{T}-[a, \sigma(a))$ and $\mathbb{T}_{\kappa}^{\kappa}:=\mathbb{T}-([a, \sigma(a)) \cup(\rho(b), b])$ which are closed. Therefore they are time scales too, and we are also able to define the above space and norm using $\mathbb{T}_{\kappa}^{\kappa}$ instead of $\mathbb{T}$.

Definition 2.1. [16] Let $f$ be a function from $\mathbb{T}$ to $\mathbb{R}$. Then, $f$ is delta differentiable at $t \in \mathbb{T}^{\kappa}$ if there exists a number $f^{\Delta}(t)$ with the following property: for any $\varepsilon>0$ there exists a $\delta>0$ such that $s \in \mathbb{T}$ and

$$
|t-s|<\delta \Rightarrow\left|f(\sigma(t))-f(s)-f^{\Delta}(t)(\sigma(t)-s)\right| \leq \varepsilon|\sigma(t)-s|
$$

Here, $f$ is said to be delta differentiable, if $f$ is delta differentiable, for all $t \in \mathbb{T}^{\kappa}$.

Definition 2.2. [16] Let $f$ be a function from $\mathbb{T}$ to $\mathbb{R}$. Then, $f$ is nabla differentiable at $t \in \mathbb{T}_{\kappa}$ if there exists a number $f^{\nabla}(t)$ with the following property: for any $\varepsilon>0$ there exists a $\delta>0$ such that $s \in \mathbb{T}$ and

$$
|t-s|<\delta \Rightarrow\left|f(\rho(t))-f(s)-f^{\nabla}(t)(\rho(t)-s)\right| \leq \varepsilon|\rho(t)-s|
$$

Here, $f$ is said to be nabla differentiable, if $f$ is nabla differentiable, for all $t \in \mathbb{T}_{\kappa}$.

Definition 2.3. [16] Let $F$ be a function from $\mathbb{T}$ to $\mathbb{R}$. Then, $F$ is called a nabla antiderivative of $f: \mathbb{T} \rightarrow \mathbb{R}$ provided $F^{\nabla}(t)=f(t)$ holds for all $t \in \mathbb{T}_{\kappa}$. Moreover, the nabla integral of $f$ is defined as follows:

$$
\int_{a}^{t} f(s) \nabla s=F(t)-F(a), \text { for all } t \in \mathbb{T}
$$

Definition 2.4. [12] Let $\mathbb{T}$ be a time scale. Then, $\mathbb{T}$ is called symmetric, if $b-t+a \in \mathbb{T}$, for all $t \in \mathbb{T}$.

Definition 2.5. [12] Let $\vartheta$ be a function from $\mathbb{T}$ to $\mathbb{R}$. If, for all $t \in \mathbb{T}, \vartheta(t)=\vartheta(b-t+a)$, then $\vartheta$ is called symmetric on $\mathbb{T}$. 
Definition 2.6. Let $\vartheta, \varphi$ be functions from $\mathbb{T}$ to $\mathbb{R}$. If a pair of $(\vartheta, \varphi)$ is a solution of $\operatorname{SSBVP}(1)-(2)$ and $\vartheta, \varphi$ are symmetric on $\mathbb{T}$, then a pair of $(\vartheta, \varphi)$ is called a symmetric solution of $S S B V P(1)-(2)$ on $\mathbb{T}$.

Definition 2.7. [19] Let $\mathbb{X}$ be a real Banach space. A nonempty closed set $P \subset \mathbb{X}$ is called a cone of $\mathbb{X}$ if it satisfies the following two conditions:

i. $y \in P, \gamma>0$ implies $\gamma y \in P$

ii. $y,-y \in P$ implies $y=0$

Theorem 2.8. $[18,19]$ Let $\mathbb{B}$ be a Banach space, and $P \subset \mathbb{B}$ is a cone in $\mathbb{B}$. Assume that $\omega_{1}$ and $\omega_{2}$ are open subsets of $\mathbb{B}$ with $0 \in \omega_{1}$ and $\overline{\omega_{1}} \subset \omega_{2}$. Let $T: P \cap\left(\overline{\omega_{2}} \backslash \omega_{1}\right) \rightarrow P$ be a completely continuous operator such that either

i. $\forall u \in P \cap \partial \omega_{1}:\|T u\| \leq\|u\|, \forall u \in P \cap \partial \omega_{2}:\|T u\| \geq\|u\|$

or

ii. $\forall u \in P \cap \partial \omega_{1}:\|T u\| \geq\|u\|, \forall u \in P \cap \partial \omega_{2}:\|T u\| \leq\|u\|$

holds. Then, $T$ has a fixed point in $P \cap\left(\overline{\omega_{2}} \backslash \omega_{1}\right)$.

Hereinafter, $\mathbb{T}$ is a symmetric time scale with $a=\min \mathbb{T}$ and $b=\max \mathbb{T}$, and $\tau_{1}, \tau_{2} \in \mathbb{T}$ such that $\tau_{1}=b-$ $\tau_{2}+a$.

Lemma 2.9. Let $q \in \mathcal{C}_{l d}(\mathbb{T})$ and $q(t) \not \equiv 0$. When $\tau_{1}, \tau_{2} \in \mathbb{T}$ such that $\tau_{1}=b-\tau_{2}+a$ and $\alpha \geq b-a$, then the BVP

$$
\begin{gathered}
\vartheta^{\Delta \nabla}(t)=-q(t), t \in \mathbb{T}_{\kappa}^{\kappa} \\
\vartheta(t)=\vartheta(b-t+a), \alpha\left[\vartheta^{\Delta}(a)-\vartheta^{\Delta}(\rho(b))\right]=\vartheta\left(\tau_{1}\right)+\vartheta\left(\tau_{2}\right)
\end{gathered}
$$

has a unique solution

$$
\vartheta(t)=\int_{a}^{b} G(t, s) q(s) \nabla s
$$

where

$$
G(t, s)=G_{1}(t, s)+G_{2}(s)
$$

here

$$
G_{1}(t, s)=\frac{1}{b-a}\left\{\begin{array}{l}
(b-t)(s-a), s \leq t \\
(b-s)(t-a), t \leq s
\end{array}\right.
$$

and

$$
G_{2}(s)=\frac{1}{2}\left\{\begin{array}{c}
\alpha+a-s, a \leq s \leq \tau_{1} \\
\alpha+\tau_{2}-b, \tau_{1} \leq s \leq \tau_{2} \\
\alpha-b+s, \tau_{2} \leq s \leq b .
\end{array}\right.
$$

Proof. Let assume that $\vartheta \in \mathcal{C}_{l d}(\mathbb{T})$ is a solution of (3)-(4). By integration of both sides of (3) from $a$ to $t$, we get

$$
\vartheta^{\Delta}(t)=\vartheta^{\Delta}(a)-\int_{a}^{t} q(s) \nabla s
$$


Integrating again, we have

$$
\vartheta(t)=\vartheta(a)+(t-a) \vartheta^{\Delta}(a)-\int_{a}^{t}(t-s) q(s) \nabla s
$$

Plug in $t=b$ in (7), we find

$$
\vartheta(b)=\vartheta(a)+(b-a) \vartheta^{\Delta}(a)-\int_{a}^{b}(b-s) q(s) \nabla s
$$

Using boundary conditions (4), we find

$$
\vartheta^{\Delta}(a)=\frac{1}{b-a} \int_{a}^{b}(b-s) q(s) \nabla s
$$

Substituting (8) to (7), we get

$$
\vartheta(t)=\vartheta(a)+(t-a) \frac{1}{b-a} \int_{a}^{b}(b-s) q(s) \nabla s-\int_{a}^{t}(t-s) q(s) \nabla s
$$

From (4), we have

$$
\begin{aligned}
\alpha \int_{a}^{\rho(\mathrm{b})} q(s) \nabla s & =2 \vartheta(a)+\left(\tau_{1}-a\right) \frac{1}{b-a} \int_{a}^{b}(b-s) q(s) \nabla s-\int_{a}^{\tau_{1}}\left(\tau_{1}-s\right) q(s) \nabla s \\
& +\left(\tau_{2}-a\right) \frac{1}{b-a} \int_{a}^{b}(b-s) q(s) \nabla s-\int_{a}^{\tau_{2}}\left(\tau_{2}-s\right) q(s) \nabla s
\end{aligned}
$$

Therefore,

$$
\begin{aligned}
\vartheta(a)= & \frac{1}{2}\left[\int_{a}^{\tau_{1}}\left[\alpha+\frac{\left(2 a-\tau_{1}-\tau_{2}\right)(b-s)}{b-a}+\left(\tau_{1}-s\right)+\left(\tau_{2}-s\right)\right] q(s) \nabla s\right. \\
& +\int_{\tau_{1}}^{\tau_{2}}\left[\alpha+\frac{\left(2 a-\tau_{1}-\tau_{2}\right)(b-s)}{b-a}+\left(\tau_{2}-s\right)\right] q(s) \nabla s \\
& \left.+\int_{\tau_{2}}^{\rho(b)}\left[\alpha+\frac{\left(2 a-\tau_{1}-\tau_{2}\right)(b-s)}{b-a}\right] q(s) \nabla s\right]
\end{aligned}
$$

and from $\tau_{1}=b-\tau_{2}+a$,

$$
\vartheta(a)=\frac{1}{2}\left[\int_{a}^{\tau_{1}}(\alpha+a-s) q(s) \nabla s+\int_{\tau_{1}}^{\tau_{2}}\left(\alpha+\tau_{2}-b\right) q(s) \nabla s+\int_{\tau_{2}}^{\rho(b)}(\alpha+s-b) q(s) \nabla s\right]
$$


Substituting (11) to (9), we have

$$
\begin{aligned}
\vartheta(t) & =\frac{1}{b-a} \int_{a}^{b}(t-a)(b-s) q(s) \nabla s-\int_{a}^{t}(t-s) q(s) \nabla s+\int_{a}^{b} G_{2}(s) q(s) \nabla s \\
& =\int_{a}^{b} G(t, s) q(s) \nabla s
\end{aligned}
$$

This proof is completed.

Lemma 2.10. For $t, s \in \mathbb{T}$, we have $G(t, s) \geq 0$ and $\min _{t \in \mathbb{T}} G_{2}(t)=G_{2}\left(\tau_{1}\right)=G_{2}\left(\tau_{2}\right)$.

Proof. It is evident from (5).

Lemma 2.11. For $t, s \in \mathbb{T}$, let $m_{G_{2}}=\min _{t \in \mathbb{T}} G_{2}(t)=G_{2}\left(\tau_{1}\right)=G_{2}\left(\tau_{2}\right)$ and $L=\frac{4 m_{G_{2}}}{4 m_{G_{2}}+(b-a)}$, then the function $G(t, s)$ satisfies

$$
L G(s, s) \leq G(t, s) \leq G(s, s) \text { for } t, s \in \mathbb{T}
$$

Proof. For $s \leq t$, we get

$$
\frac{G_{1}(t, s)}{G_{1}(s, s)}=\frac{b-t}{b-s} \leq \frac{b-s}{b-s}=1
$$

For $t \leq s$, we get

$$
\frac{G_{1}(t, s)}{G_{1}(s, s)}=\frac{t-a}{s-a} \leq \frac{s-a}{s-a}=1
$$

Therefore, we have $G_{1}(t, s) \leq G_{1}(s, s)$. Thus,

$$
\mathrm{G}(\mathrm{t}, \mathrm{s})=G_{1}(t, s)+G_{2}(s) \leq G_{1}(s, s)+G_{2}(s)=G(s, s)
$$

We know $G_{2}(s) \geq 0$ from Lemma 2.9. Therefore, it is evident that $m_{G_{2}} \geq 0$. By using $\frac{(b-s)(s-a)}{b-a} \leq \frac{b-a}{4}$, we have

$$
\begin{aligned}
G(t, s) & =G_{1}(t, s)+G_{2}(s) \\
& \geq G_{2}(s) \\
& =\frac{4 m_{G_{2}}+b-a}{4 m_{G_{2}}+b-a} G_{2}(s) \\
& \geq L G_{2}(s)+\frac{4}{4} \frac{(b-a) \cdot m_{G_{2}}}{4 m_{G_{2}}+b-a} \\
& =L G_{2}(s)+L \frac{(b-a)}{4} \\
& \geq L G_{2}(s)+L \frac{(b-s)(s-a)}{b-a} \\
& =L G_{2}(s)+L G_{1}(s, s) \\
& =L G(s, s)
\end{aligned}
$$

It is evident that $L G(s, s) \leq G(t, s) \leq G(s, s)$ for $t, s \in \mathbb{T}$. This proof is completed. 
Lemma 2.12. $G(t, s)$ Green function is symmetric on $\mathbb{T}$. i.e., for $t, s \in \mathbb{T}$,

$$
G(b-t+a, b-s+a)=G(t, s)
$$

Proof. Using $\tau_{1}=b-\tau_{2}+a$ and Definition 2.5 and (5), we have

$$
\begin{aligned}
G_{1}(b-t+a, b-s+a) & =\frac{1}{b-a}\left\{\begin{array}{l}
(b-b+t-a)(b-s+a-a), b-s+a \leq b-t+a \\
(b-b+s-a)(b-t+a-a), b-t+a \leq b-s+a
\end{array}\right. \\
& =\frac{1}{b-a}\left\{\begin{array}{l}
(t-a)(b-s), t \leq s \\
(s-a)(b-t), s \leq t
\end{array}\right. \\
& =G_{1}(t, s)
\end{aligned}
$$

and

$$
\begin{aligned}
G_{2}(b-s+a)= & \frac{1}{2}\left\{\begin{array}{c}
\alpha+a-b+s-a, a \leq b-s+a \leq \tau_{1} \\
\alpha+\tau_{2}-b, \tau_{1} \leq b-s+a \leq \tau_{2} \\
\alpha-b+b-s+a, \tau_{2} \leq b-s+a \leq b
\end{array}\right. \\
= & \frac{1}{2}\left\{\begin{array}{c}
\alpha-b+s, a-b-a \leq-s \leq \tau_{1}-b-a \\
\alpha+\tau_{2}-b, \tau_{1}-b-a \leq-s \leq \tau_{2}-b-a \\
\alpha+a-s, \tau_{2}-b-a \leq-s \leq b-b-a
\end{array}\right. \\
= & \frac{1}{2}\left\{\begin{array}{c}
\alpha-b+s, \tau_{2} \leq s \leq b \\
\alpha+\tau_{2}-b, \tau_{1} \leq s \leq \tau_{2} \\
\alpha+a-s, a \leq s \leq \tau_{1}
\end{array}\right. \\
= & G_{2}(s)
\end{aligned}
$$

Therefore,

$$
G(b-t+a, b-s+a)=G_{1}(b-t+a, b-s+a)+G_{2}(b-s+a)=G_{1}(t, s)+G_{2}(s)
$$

Eventually, for $t, s \in \mathbb{T}, G(b-t+a, b-s+a)=G(t, s)$. The proof is completed.

\section{Main Results}

This section studies the existence of the SPSs of the SSBVP (1)-(2). First, we assume the following conditions: $\left(H_{1}\right) f: \mathbb{T} \times \mathbb{R}^{+} \rightarrow \mathbb{R}^{+}$is a ld-continuous function such that $f(., s)$ is symmetric on $\mathbb{T}$ and $f(t, 0) \equiv 0$, $\left(H_{2}\right) g: \mathbb{T} \times \mathbb{R}^{+} \rightarrow \mathbb{R}^{+}$is a ld-continuous function such that $g(., s)$ is symmetric on $\mathbb{T}$ and $g(t, 0) \equiv 0$.

Second, we give the following assumptions:

$$
\begin{array}{ll}
\overline{f_{0}}=\overline{\lim }_{x \rightarrow 0^{+}} \max _{t \in \mathbb{T}} \frac{f(t, x)}{x}, & \overline{g_{0}}=\overline{\lim }_{x \rightarrow 0^{+}} \max _{t \in \mathbb{T}} \frac{g(t, x)}{x}, \\
\underline{f_{0}}=\lim _{x \rightarrow 0^{+}} \min _{t \in \mathbb{T}} \frac{f(t, x)}{x}, & \underline{g_{0}}=\lim _{x \rightarrow 0^{+}} \min _{t \in \mathbb{T}} \frac{g(t, x)}{x}, \\
\overline{f_{\infty}}=\overline{\lim }_{x \rightarrow \infty} \max _{t \in \mathbb{T}} \frac{f(t, x)}{x}, & \overline{g_{\infty}}=\overline{\lim }_{x \rightarrow \infty} \max _{t \in \mathbb{T}} \frac{g(t, x)}{x}, \\
\underline{f_{\infty}}=\lim _{\underline{x \rightarrow \infty}} \min _{t \in \mathbb{T}} \frac{f(t, x)}{x}, & \underline{g_{\infty}}=\lim _{x \rightarrow \infty} \min _{t \in \mathbb{T}} \frac{g(t, x)}{x}
\end{array}
$$

Now, let $\mathbb{B}=\mathcal{C}_{l d}(\mathbb{T})$ be a Banach space with $\|\vartheta\|=\max _{t \in \mathbb{T}}|\vartheta(t)|$, and determine cone $P \subset \mathbb{B}$ by

$$
P=\left\{\vartheta \in \mathbb{B} \mid \vartheta(t) \geq 0 \text { for } t \in \mathbb{T}, \vartheta(t) \text { is symmetric on } \mathbb{T} \text {, } \min _{t \in \mathbb{T}} \vartheta(t) \geq L\|\vartheta\| \text { and } \vartheta^{\Delta \nabla}(t) \leq 0 \text { for } t \in \mathbb{T}_{\kappa}^{\kappa}\right\}
$$


Besides, define the integral operator $T$ from $P$ to $\mathbb{B}$ by

$$
T \vartheta(t)=\int_{a}^{b} G(t, s) f\left(s, \int_{a}^{b} G(s, \zeta) g(\zeta, \vartheta(\zeta)) \nabla \zeta\right) \nabla s
$$

Thus,

$$
T \vartheta(t)^{\Delta \nabla}=-f\left(s, \int_{a}^{b} G(s, \zeta) g(\zeta, \vartheta(\zeta)) \nabla \zeta\right)
$$

Hence, for $\vartheta \in P, T \vartheta \geq 0$ on $\mathbb{T}$ and $T \vartheta^{\Delta \nabla} \leq 0$ on $\mathbb{T}_{\kappa}^{\kappa}$.

Since $\vartheta, f$ and $g$ are symmetric on $\mathbb{T}$, then

$$
\begin{aligned}
T \vartheta(b-t+a) & =\int_{b}^{a} G(b-t+a, b-s+a) f\left(b-s+a, \int_{a}^{b} G(b-s+a, \zeta) g(\zeta, \vartheta(\zeta)) \nabla \zeta\right) \nabla(b-s+a) \\
& =\int_{a}^{b} G(t, s) f\left(b-s+a, \int_{b}^{a} G(b-s+a, b-\zeta+a) g(b-\zeta+a, \vartheta(b-\zeta+a)) \nabla(b-\zeta+a)\right) \nabla s \\
& =\int_{a}^{b} G(t, s) f\left(s, \int_{a}^{b} G(s, \zeta) g(\zeta, \vartheta(\zeta)) \nabla \zeta\right) \nabla s \\
& =T \vartheta(t)
\end{aligned}
$$

for all $t \in \mathbb{T}$. This implies that $T \vartheta(t)$ is symmetric on $\mathbb{T}$. It is easy to verify that $\min _{t \in \mathbb{T}} T \vartheta(t) \geq L\|T \vartheta\|$. Consequently $T: P \rightarrow P$.

Lemma 3.1. Suppose that $\left(H_{1}\right)$ and $\left(H_{2}\right)$ hold. Then, for $\vartheta, \varphi \in \mathbb{B}$, a pair of $(\vartheta, \varphi)$ is a solution of SSBVP (1)-(2) iff $\vartheta$ is a fixed point of the operator $T$ and $\varphi(\mathrm{t})=\int_{a}^{b} G(t, \zeta) g(\zeta, \vartheta(\zeta)) \nabla \zeta$

Proof. The proof is clear from Lemma 2.9.

Lemma 3.2. Suppose that $\left(H_{1}\right)$ and $\left(H_{2}\right)$ hold. Then, the operator $T: P \rightarrow P$ is completely continuous.

Proof. Suppose that $K \subset P$ is a bounded set. Let $N \geq 0$ be such that $\|\vartheta\| \leq N$ for $\vartheta \in K$, we have

$$
\begin{aligned}
|T \vartheta(t)| & \leq \int_{a}^{b} G(s, s) f\left(s, \int_{a}^{b} G(s, \zeta) g(\zeta, \vartheta(\zeta)) \nabla \zeta\right) \nabla s \\
& \leq \int_{a}^{b} G(s, s) \sup _{a<s<b}\left|f\left(s, \int_{a}^{b} G(s, \zeta) g(\zeta, \vartheta(\zeta)) \nabla \zeta\right)\right| \nabla s \\
& =\sup _{a<s<b}\left|f\left(s, \int_{a}^{b} G(s, \zeta) g(\zeta, \vartheta(\zeta)) \nabla \zeta\right)\right| \int_{a}^{b} G(s, s) \nabla s
\end{aligned}
$$

for every $t \in \mathbb{T}$. This implies that $T(K)$ is bounded. By the Arzela-Ascoli theorem, we can easily see that $T$ is a completely continuous operator. Thus, the proof is completed.

For convenience, we denote

$$
m:=\int_{a}^{b} G(z, z) \nabla z
$$


Theorem 3.3. Suppose that $\left(H_{1}\right)$ and $\left(H_{2}\right)$ are satisfied. If $\overline{f_{0}}=\overline{g_{0}}=0$ and $\underline{f_{\infty}}=\underline{g_{\infty}}=\infty$ hold, then SSBVP (1)-(2) has a symmetric positive solution $(\vartheta, \varphi)$.

Proof. Because of $\overline{f_{0}}=\overline{g_{0}}=0$ uniformly on $\mathbb{T}$, we may choose a $c_{1}>0$ such that

$$
f(t, \vartheta) \leq \gamma_{1} \vartheta, \quad g(t, \vartheta) \leq \gamma_{1} \vartheta, \quad 0<\vartheta \leq c_{1}, \quad t \in \mathbb{T}
$$

where $\gamma_{1} \leq \frac{1}{m}$. Note that

$$
\int_{a}^{b} G(s, \zeta) g(\zeta, \vartheta(\zeta)) \nabla \zeta \leq \int_{a}^{b} G(\zeta, \zeta) \gamma_{1} \vartheta(\zeta) \nabla \zeta \leq \gamma_{1} \int_{a}^{b} G(\zeta, \zeta)\|\vartheta\| \nabla \zeta \leq\|\vartheta\| \leq c_{1}
$$

If $\omega_{1}$ is a ball in $\mathbb{B}$ centred at the origin with a radius $c_{1}$ and if $\vartheta \in P \cap \partial \omega_{1}$, then we have

$$
\begin{aligned}
\|T \vartheta(t)\| & =\max _{t \in \mathbb{T}} \int_{a}^{b} G(t, s) f\left(s, \int_{a}^{b} G(s, \zeta) g(\zeta, \vartheta(\zeta)) \nabla \zeta\right) \nabla s \\
& \leq \int_{a}^{b} G(s, s) \gamma_{1} \int_{a}^{b} G(s, \zeta) g(\zeta, \vartheta(\zeta)) \nabla \zeta \nabla s \\
& \leq \gamma_{1} \int_{a}^{b} G(s, s) \nabla s \int_{a}^{b} G(\zeta, \zeta) \gamma_{1} \vartheta(\zeta) \nabla \zeta \\
& \leq \gamma_{1}{ }^{2} \int_{a}^{b} G(s, s) \nabla s \int_{a}^{b} G(\zeta, \zeta)\|\vartheta\| \nabla \zeta \\
& =\gamma_{1}{ }^{2} m^{2}\|\vartheta\| \leq\|\vartheta\|
\end{aligned}
$$

and so $\|T \vartheta(t)\| \leq\|\vartheta\|$ for all $\vartheta \in P \cap \partial \omega_{1}$.

Next, we use the assumption $\underline{f_{\infty}}=\underline{g_{\infty}}=\infty$ uniformly on $\mathbb{T}$. There exists a $c_{2}>0$ large enough such that $f(t, \vartheta) \geq \mu \vartheta, g(t, \vartheta) \geq \mu \vartheta, \vartheta>c_{2}$, for $t \in \mathbb{T}$ where $\mu \geq \frac{1}{L^{\frac{3}{2}} m}$. If we define $\omega_{2}=\left\{\vartheta \in \mathbb{B} \mid\|\vartheta\|<\frac{2 c_{2}}{\sqrt{L}}\right\}$, for $t \in \mathbb{T}, \vartheta \in P$ and $\|\vartheta\|=\frac{2 c_{2}}{\sqrt{L}}$, we have

$$
\int_{a}^{b} G(s, \zeta) g(\zeta, \vartheta(\zeta)) \nabla \zeta \geq L \int_{a}^{b} G(\zeta, \zeta) \mu \vartheta(\zeta) \nabla \zeta \geq L \mu \int_{a}^{b} G(\zeta, \zeta) \nabla \zeta L\|\vartheta\|=L^{2} \mu m\|\vartheta\| \geq \sqrt{L}\|\vartheta\|=2 c_{2}>c_{2}
$$

For $\vartheta \in P \cap \partial \omega_{2}$, we have

$$
\begin{aligned}
\|T \vartheta(t)\| & \geq L \int_{a}^{b} G(s, s) \mu \int_{a}^{b} G(s, \zeta) g(\zeta, \vartheta(\zeta)) \nabla \zeta \nabla s \\
& \geq L^{2} \mu \int_{a}^{b} G(s, s) \nabla s \int_{a}^{b} G(\zeta, \zeta) \mu \vartheta(\zeta) \nabla \zeta \\
& \geq L^{2} \mu^{2} \int_{a}^{b} G(s, s) \nabla s \int_{a}^{b} G(\zeta, \zeta) L\|\vartheta\| \nabla \zeta \\
& =\mu^{2} L^{3} m^{2}\|\vartheta\| \geq\|\vartheta\|
\end{aligned}
$$


and so $\|T \vartheta(t)\| \geq\|\vartheta\|$ for all $\vartheta \in P \cap \partial \omega_{2}$. As a conclusion, from (i) of Theorem 2.8, $T$ has a fixed point in $P \cap\left(\overline{\omega_{2}} \backslash \omega_{1}\right)$ and so the $\operatorname{SSBVP}(1)-(2)$ has a symmetric positive solution $(\vartheta, \varphi)$.

Theorem 3.4. Assume $\left(H_{1}\right)$ and $\left(H_{2}\right)$ are satisfied. If $\underline{f_{0}}=\underline{g_{0}}=\infty$ and $\overline{f_{\infty}}=\overline{g_{\infty}}=0$ hold, then SSBVP (1)(2) has a symmetric positive solution $(\vartheta, \varphi)$.

PROof. Firstly, let $\underline{f_{0}}=\underline{g_{0}}=\infty$ hold. For $t \in \mathbb{T}$, there exists a $\overline{c_{3}}>0$ such that

$$
f(t, \vartheta) \geq \mu \vartheta, g(t, \vartheta) \geq \mu \vartheta, 0<\vartheta<\overline{c_{3}}
$$

where $\mu \geq \frac{1}{L^{\frac{3}{2} m}}$. Now from $g(t, 0) \equiv 0$ and $g(t, s)$ is continuous, we know that there exists a number $c_{3} \in$ $\left(0, \overline{c_{3}}\right)$ such that $g(t, \vartheta) \leq \frac{\overline{c_{3}}}{m}$ for each $\vartheta \in\left(0, \overline{c_{3}}\right]$ and $t \in \mathbb{T}$. Then, for all $\vartheta \in P$ and $\|\vartheta\|=\overline{c_{3}}$, note that

$$
\int_{a}^{b} G(s, \zeta) g(\zeta, \vartheta(\zeta)) \nabla \zeta \leq \int_{a}^{b} G(\zeta, \zeta) \frac{\overline{c_{3}}}{m} \nabla \zeta=\overline{c_{3}}
$$

Let $\omega_{3}=\left\{\vartheta \in \mathbb{B} \mid\|\vartheta\|<c_{3}\right\}$. For $\vartheta \in P \cap \partial \omega_{3}$, then we have

$$
\begin{aligned}
\|T \vartheta(t)\| & \geq L \int_{a}^{b} G(s, s) \mu \int_{a}^{b} G(s, \zeta) g(\zeta, \vartheta(\zeta)) \nabla \zeta \nabla s \\
& \geq L^{2} \mu \int_{a}^{b} G(s, s) \nabla s \int_{a}^{b} G(\zeta, \zeta) \mu \vartheta(\zeta) \nabla \zeta \\
& \geq L^{2} \mu^{2} \int_{a}^{b} G(s, s) \nabla s \int_{a}^{b} G(\zeta, \zeta) L\|\vartheta\| \nabla \zeta \geq\|\vartheta\|
\end{aligned}
$$

For $\vartheta \in P \cap \partial \omega_{3}$, we have $\|T \vartheta(t)\| \geq\|\vartheta\|$. Next, since $\overline{f_{\infty}}=\overline{g_{\infty}}=0$, there exists a $c_{4}>0$ such that

$$
f(t, \vartheta) \leq \gamma_{4} \vartheta, \quad g(t, \vartheta) \leq \gamma_{4} \vartheta, \quad \vartheta>c_{4}, \quad t \in \mathbb{T}
$$

where $\gamma_{4} \leq \frac{1}{m}$. We consider two cases.

Case 1. Assume $f(t, s)$ is bounded on $\mathbb{T} \times[0, \infty)$. Therefore, there is an $M>0$ such that $f(t, s) \leq M$, for $t \in$ $\mathbb{T}$ and $s \in[0, \infty)$. Let $c_{4}{ }^{*} \geq \max \left\{2 c_{4}, M m\right\}$. Then, for $\vartheta \in P$ with $\|\vartheta\|=c_{4}{ }^{*}$,

$$
\|T \vartheta(t)\| \leq \int_{a}^{b} G(s, s) M \nabla s=M m \leq \max \left\{2 c_{4}, M m\right\} \leq{c_{4}}^{*}=\|\vartheta\|
$$

Case 2. Assume $f(t, s)$ is unbounded on $\mathbb{T} \times[0, \infty)$. Then,

$$
h(c):=\max \{f(t, s) \mid t \in \mathbb{T}, 0 \leq s \leq c\}
$$

such that $\lim _{c \rightarrow \infty} h(c)=\infty$. Therefore, we can choose $c_{4}{ }^{*} \geq \max \left\{2 c, c_{4}\right\}$ such that $h(c) \leq h\left(c_{4}{ }^{*}\right)$ for $0 \leq c \leq$ $c_{4}{ }^{*}$. Since $c_{4} \leq c_{4}{ }^{*},(15)$ and (16), then we get $f(t, s) \leq h\left(c_{4}{ }^{*}\right) \leq \gamma_{4} c_{4}{ }^{*}$, for $t \in \mathbb{T}$ and $s \in\left[0, c_{4}{ }^{*}\right]$. For $\vartheta \in$ $P$ and $\|\vartheta\|=c_{4}{ }^{*}$, we have

$$
\|T \vartheta(t)\| \leq \int_{a}^{b} G(s, s) \gamma_{4} c_{4}{ }^{*} \nabla s=\gamma_{4} c_{4}{ }^{*} m \leq c_{4}{ }^{*}=\|\vartheta\|
$$


So, we obtain $\|T \vartheta(t)\| \leq\|\vartheta\|$ for all $\vartheta \in P \cap \partial \omega_{4}$, where $\omega_{4}=\left\{\vartheta \in \mathbb{B} \mid\|\vartheta\| \leq c_{4}{ }^{*}\right\}$ in both cases. By (ii) of Theorem 2.8 that $T$ has a fixed point in $P \cap\left(\overline{\omega_{4}} \backslash \omega_{3}\right)$ and so the SSBVP (1)-(2) has a symmetric positive solution $(\vartheta, \varphi)$.

We will provide sufficient conditions for two SPSs for SSBVP (1)-(2).

$\left(H_{3}\right)$ There exists a constant $R_{1}>0$ such that $f(t, s) \leq \frac{R_{1}}{m}$ and $g(t, s) \leq \frac{R_{1}}{m}$, for $t \in \mathbb{T}, s \in\left[0, R_{1}\right]$.

$\left(H_{4}\right)$ There exists a constant $R_{2}>0$ such that $f(t, s) \geq \frac{R_{2}}{L m}$ and $g(t, s) \leq \frac{R_{2}}{m}$, for $t \in \mathbb{T}, s \in\left[0, R_{2}\right]$.

Theorem 3.5. Assume that $\left(H_{1}\right),\left(H_{2}\right)$ and $\left(H_{3}\right)$ are satisfied. If $\underline{f_{\infty}}=\underline{g_{\infty}}=\infty$ and $\underline{f_{0}}=\underline{g_{0}}=\infty$ hold, then the $\operatorname{SSBVP}(1)-(2)$ has two SPSs $\left(\vartheta_{1}, \varphi_{1}\right)$ and $\left(\vartheta_{2}, \varphi_{2}\right)$.

ProOF. At first, from Lemma 2.11 and $\left(H_{3}\right)$, we can obtain $\int_{a}^{b} G(s, \zeta) g\left(\zeta, \vartheta(\zeta) \nabla \zeta \in\left[0, R_{1}\right]\right.$. Thus $\|T \vartheta(t)\| \leq$ $\frac{R_{1}}{m} \int_{a}^{b} G(s, s) \nabla s=R_{1}=\|\vartheta\|$. Then $\|T \vartheta(t)\| \leq\|\vartheta\|$ for $\forall \vartheta \in P \cap \partial \omega_{5}$, where $\omega_{5}=\left\{\vartheta \in \mathbb{B} \mid\|\vartheta\|<R_{1}\right\}$. For another hand, from Theorem 3.3 and Theorem 3.4, we have $\|T \vartheta(t)\| \geq\|\vartheta\|$ for $\forall \vartheta \in P \cap \partial \omega_{2}$ where $c_{2}>$ $R_{1}$ and $\|T \vartheta(t)\| \geq\|\vartheta\|$ for $\forall \vartheta \in P \cap \partial \omega_{3}$ where $R_{1}>c_{3}$.

It follows from Theorem 2.8 that $T$ has a fixed point $\vartheta_{1}$ in $P \cap\left(\overline{\omega_{5}} \backslash \omega_{3}\right)$ and a fixed point $\vartheta_{2}$ in $P \cap$ $\left(\overline{\omega_{2}} \backslash \omega_{5}\right) .\left(\vartheta_{1}, \varphi_{1}\right)$ and $\left(\vartheta_{2}, \varphi_{2}\right)$ are SPSs of the SSBVP $(1)-(2)$.

Theorem 3.6. Assume that $\left(H_{1}\right),\left(H_{2}\right)$ and $\left(H_{4}\right)$ are satisfied. If $\overline{f_{0}}=\overline{g_{0}}=0$ and $\overline{f_{\infty}}=\overline{g_{\infty}}=0$ hold, then the SSBVP (1)-(2) has two SPSs.

PRoOF. It could be proved in a similar way to Theorems 3.5.

Example 3.7. On a bounded symmetric time scale $\mathbb{T}=\{1,2,3,4,5,6,7,8,9,10\}$ we consider following SSBVP

$$
\begin{array}{ll}
\vartheta^{\Delta \nabla}(t)=-\varphi^{2}(t)\left(\frac{11}{2}-t\right)^{2}, & t \in \mathbb{T}_{\kappa}^{\kappa}=\{2,3,4,5,6,7,8,9\} \\
\varphi^{\Delta \nabla}(t)=-\vartheta^{4}(t)\left(\frac{11}{2}-t\right)^{2}, & t \in \mathbb{T}_{\kappa}^{\kappa}=\{2,3,4,5,6,7,8,9\}
\end{array}
$$

and boundary conditions

$$
\begin{aligned}
\vartheta(t)=\vartheta(11-t), & 9\left(\vartheta^{\Delta}(1)-\vartheta^{\Delta}(9)\right)=\vartheta(3)+\vartheta(8) \\
\varphi(t)=\varphi(11-t), & 9\left(\varphi^{\Delta}(1)-\varphi^{\Delta}(9)\right)=\varphi(3)+\varphi(8)
\end{aligned}
$$

In this problem, $[1,10]_{\mathbb{T}}$ is symmetric, $a=1, b=10, \sigma(a)=2, \rho(b)=9, \tau_{1}=3$ and $\tau_{2}=8$. We see that easily $f(t, \varphi)=\left(\frac{11}{2}-t\right)^{2} \varphi^{2}$ and $g(t, \vartheta)=\left(\frac{11}{2}-t\right)^{2} \vartheta^{4}$ are satisfies the conditions $\left(H_{1}\right)$ and $\left(H_{2}\right)$ and also

$$
\begin{aligned}
& \overline{f_{0}}=\overline{\lim _{\varphi \rightarrow 0^{+}}} \max _{t \in \mathbb{T}} \frac{f(t, \varphi)}{\varphi}=\overline{\lim _{\varphi \rightarrow 0^{+}}} \max _{t \in \mathbb{T}}\left(\frac{11}{2}-t\right)^{2} \varphi=0 \\
& \overline{g_{0}}=\overline{\lim _{\vartheta \rightarrow 0^{+}}} \max _{t \in \mathbb{T}} \frac{g(t, \vartheta)}{\vartheta}=\overline{\lim _{\vartheta \rightarrow 0^{+}}} \max _{t \in \mathbb{T}}\left(\frac{11}{2}-t\right)^{2} \vartheta^{3}=0 \\
& \underline{f_{\infty}}=\underline{\lim _{\varphi \rightarrow \infty}} \min _{t \in \mathbb{T}} \frac{f(t, \varphi)}{\varphi}=\underline{\lim _{\varphi \rightarrow \infty}} \min _{t \in \mathbb{T}}\left(\frac{11}{2}-t\right)^{2} \varphi=\infty \\
& \underline{g_{\infty}}=\underline{\lim _{\vartheta \rightarrow \infty}} \min _{t \in \mathbb{T}} \frac{g(t, \vartheta)}{\vartheta}=\underline{\lim _{\vartheta \rightarrow \infty}} \min _{t \in \mathbb{T}}\left(\frac{11}{2}-t\right)^{2} \vartheta^{3}=\infty
\end{aligned}
$$

As a result, all conditions of Theorem 3.3 are satisfied. From Theorem 3.3, SSBVP (17)-(18) has one SPS.

Example 3.8. On a bounded symmetric time scale $\mathbb{T}=\{0\} \cup[1,2] \cup\{3\}$ we consider the following system 


$$
\left\{\begin{array}{l}
\vartheta^{\Delta \nabla}(t)=-f(t, \varphi(t)), t \in \mathbb{T}_{\kappa}^{\kappa}=[1,2] \\
\varphi^{\Delta \nabla}(t)=-g(t, \vartheta(t)), t \in \mathbb{T}_{\kappa}^{\kappa}=[1,2]
\end{array}\right.
$$

with boundary conditions

$$
\left\{\begin{array}{cl}
\vartheta(t)=\vartheta(3-t), & 3\left(\vartheta^{\Delta}(0)-\vartheta^{\Delta}(2)\right)=\vartheta(1)+\vartheta(2) \\
\varphi(t)=\varphi(3-t), & 3\left(\varphi^{\Delta}(0)-\varphi^{\Delta}(2)\right)=\varphi(1)+\varphi(2)
\end{array}\right.
$$

where $f(t, \varphi)=\frac{\left(\left|t-\frac{3}{2}\right|+1\right)\left(\sqrt{\varphi}+\varphi^{2}\right)}{440}$ and $g(t, \vartheta)=\frac{\left(\left|t-\frac{3}{2}\right|+1\right)\left(2 \sqrt{\vartheta}+\vartheta^{2}\right)}{880}$. Here $a=0, b=3, \sigma(a)=1, \rho(b)=2$, $\tau_{1}=1, \tau_{2}=2$ and $[0,3]_{\mathbb{T}}$ is symmetric. We see that easily $f(t, \varphi)$ and $g(t, \vartheta)$ are satisfies the conditions $\left(H_{1}\right)$ and $\left(H_{2}\right)$. Furthermore, we find

$$
\begin{aligned}
& \underline{f_{0}}=\lim _{\underline{\varphi \rightarrow 0^{+}}} \min _{t \in \mathbb{T}} \frac{f(t, \varphi)}{\varphi}=\lim _{\varphi \rightarrow 0^{+}} \min _{t \in \mathbb{T}} \frac{\left(\left|t-\frac{3}{2}\right|+1\right)\left(\sqrt{\varphi}+\varphi^{2}\right)}{440 \varphi}=\infty \\
& \underline{g_{0}}=\lim _{\underline{\vartheta \rightarrow 0^{+}}} \min _{t \in \mathbb{T}} \frac{g(t, \vartheta)}{\vartheta}=\lim _{\underline{\vartheta \rightarrow 0^{+}}} \min _{t \in \mathbb{T}} \frac{\left(\left|t-\frac{3}{2}\right|+1\right)\left(2 \sqrt{\vartheta}+\vartheta^{2}\right)}{880 \vartheta}=\infty \\
& \underline{f_{\infty}}=\lim _{\varphi \rightarrow \infty} \min _{t \in \mathbb{T}} \frac{f(t, \varphi)}{\varphi}=\lim _{\varphi \rightarrow \infty} \min _{t \in \mathbb{T}} \frac{\left(\left|t-\frac{3}{2}\right|+1\right)\left(\sqrt{\varphi}+\varphi^{2}\right)}{440 \omega}=\infty \\
& \underline{g_{\infty}}=\lim _{\underline{\vartheta \rightarrow \infty}} \min _{t \in \mathbb{T}} \frac{g(t, \vartheta)}{\vartheta}=\lim _{\underline{\vartheta \rightarrow \infty}} \min _{t \in \mathbb{T}} \frac{\left(\left|t-\frac{3}{2}\right|+1\right)\left(2 \sqrt{\vartheta}+\vartheta^{2}\right)}{880 \vartheta}=\infty
\end{aligned}
$$

We calculate

$$
m=\int_{0}^{3} G(s, s) \nabla s=\frac{44}{9}
$$

If we choose $R_{1}=2$, then we have $\frac{R_{1}}{m}=0.409$ and $f(t, s) \leq \frac{R_{1}}{m}$ and $g(t, s) \leq \frac{R_{1}}{m}$ for $t \in \mathbb{T}, s \in\left[0, R_{1}\right]$. So, $f$ and $g$ satisfy the condition $\left(H_{3}\right)$. Consequently, all conditions of Theorem 3.5 are satisfied. From Theorem 3.5, SSBVP (19)-(20) has at least two SPSs.

\section{Conclusion}

In this study, we obtain sufficient conditions that guarantee at least one and two SPSs of the system (1)-(2) on a symmetric time scale. This paper generalizes Qu's study in 2009 [6], which is the existence of SPSs of second-order differential equation systems with four-point boundary conditions to dynamic equation systems on symmetric time scales. To investigate the symmetric solutions of dynamic equations on time scales, researchers use Definition 2.4. A time scale that is symmetric in the sense of Definition 2.4 must satisfy $b-$ $t+a \in \mathbb{T}$, for all $t \in \mathbb{T}$. Because of this $\overline{q^{\mathbb{Z}}}$ is not symmetric where $q>1$. Therefore, this definition does not generalize all time scales. If a new symmetric definition can be found, including the q-difference time scales, it will be more general. Also, in the future someone can work on this problem for existence of one, two and three symmetric positive solutions by using Schauder fixed point theorem, Avery-Anderson-Henderson fixed point theorem, Legget-Williams fixed point theorem. Furthermore, this boundary values problem can be considered with impulsive boundary conditions. 


\section{Author Contributions}

All the authors contributed equally to this work. They all read and approved the last version of the manuscript.

\section{Conflict of Interest}

The authors declare no conflict of interest.

\section{References}

[1] S. Hilger, Ein Masskettenkalkül mit Anwendug auf zentrumsmanningfaltigkeiten, PhD Dissertation, Universitat Würzburg (1988) Germany.

[2] F. M. Atıc1, D. C. Biles, A. Lebedinsky, An Application of Time Scales to Economics, Mathematical and Computer Modelling 43 (2006) 718-726.

[3] M. A. Jones, B. Song, D. M. Thomas, Controlling Wound Healing Through Debridement, Mathematical and Computer Modelling 40 (2004) 1057-1064.

[4] V. Spedding, Taming Nature's Numbers, New Scientist (2003) 28-32.

[5] D. M. Thomas, L. Vandemuelebroeke, K. Yamaguchi, A Mathematical Evolution Model for Phytoremediation of Metals, Discrete and Continuous Dynamical Systems Series B 5(2) (2005) 411-422.

[6] H. Qu, The Symmetric Positive Solutions of Four-point Problems for Nonlinear Boundary Value Secondorder Differential Equations, International Journal of Mathematical Analysis, 3(40) (2009) 1969-1979.

[7] J. Henderson and H. B. Thompson, Multiple Symmetric Positive Solutions for a Second Order Boundary Value Problem, Proceedings of the American Mathematical Society 128 (2000) 2373-2379.

[8] F. Li, Y. Zhang, Multiple Symmetric Nonnegative Solutions of Second-order Ordinary Differential Equations, Applied Mathematics Letters 17 (2004) 261-267.

[9] Q. Yao, Existence and Iteration of n Symmetric Positive Solutions for a Singular Two-point Boundary Value Problem, Computers \& Mathematics with Applications 47 (2004) 1195-1200.

[10] Y. P. Sun, Existence and Multiplicity of Symmetric Positive Solutions for Three-point Boundary Value Problem, Applied Mathematics Letters 329 (2007) 998-1009.

[11] N. A. Hamal, F. Yoruk, Symmetric Positive Solutions of Fourth Order Integral BVP for an Increasing Homeomorphism and Homomorphism with Sign-changing Nonlinearity on Time Scales, Computers \& Mathematics with Applications 59(11) (2010) 3603-3611.

[12] E. Cetin, F. S. Topal, Symmetric Positive Solutions of Fourth Order Boundary Value Problems for an Increasing Homeomorphism and Homomorphism on Time Scales, Computers and Mathematics with Applications, 63(3) (2012) 669-678.

[13] K. R. Prasad, A. K. Rao and B. Bharathi, Positive Solutions for System of 2n-th Order Sturm-Liouville Boundary Value Problems on Time Scales, Proceedings of the Indian Academy of Sciences: Mathematical Sciences 124 (2014), 67-79.

[14] A. D. Oğuz, F. S. Topal, Symmetric Positive Solutions for the Systems of Higher-order Boundary Value Problems on Time Scales, Advances in Pure and Applied Mathematics 8(4) (2017) 285-292.

[15] M. Bohner, A. Peterson, Dynamic Equations on Time Scales, An Introduction with Applications, Birkhäuser, Boston, 2001. 
[16] M. Bohner, A. Peterson, (Eds.), Advances in Dynamic Equations on Time Scales, Birkhäuser, Boston, 2003.

[17] S. Hilger, Analysis on Measure Chains-A Unified Approach to Continuous and Discrete Calculus, Results in Mathematics 18 (1990) 18-56.

[18] M. Krasnosel'skii, Positive Solutions of Operator Equations, Noordhoff, Groningen, 1964.

[19] F. Deimling, Nonlinear Functional Analysis, Springer, NewYork, 1985. 